\title{
Correction to: Perioperative antibiotic prescribing in surgery departments of two private sector hospitals in Madhya Pradesh, India
}

Anna Machowska ${ }^{1 \dagger}$, Jonatan Sparrentoft ${ }^{1 \dagger}$, Shyam Kumar Dhakaita ${ }^{3}$, Cecilia StålsbyLundborg ${ }^{1 \dagger}$ and Megha Sharma ${ }^{1,2^{*}+}$

Correction to: Perioper Med (2019) 8:10

https://doi.org/10.1186/s13741-019-0121-3

Following publication of the original article (Machowska et al. 2019), the authors provided new footnotes for Table 1 and Table 2. The complete tables and updated footnotes are supplied below.

\section{Author details}

'Department of Public Health Sciences, Global Health - Health Systems and Policy, Karolinska Institutet, 17177 Stockholm, Sweden. ${ }^{2}$ Department of Pharmacology, Ruxmaniben Deepchand Gardi Medical College, Surasa, Ujjain 456006, India. ${ }^{3}$ Department of Surgery, Ruxmaniben Deepchand Gardi

Medical College, Surasa, Ujjain 456006, India.

Published online: 28 October 2019

\section{Reference}

Machowska, et al. Perioperative antibiotic prescribing in surgery departments of two private sector hospitals in Madhya Pradesh, India. Perioper Med. 2019;8: 10. https://doi.org/10.1186/s13741-019-0121-3.

\footnotetext{
The original article can be found online at https://doi.org/10.1186/s13741019-0121-3

* Correspondence: meghasharma27@rediffmail.com

${ }^{+}$Anna Machowska and Jonatan Sparrentoft contributed equally for this study and share the first authorship.

${ }^{+}$Cecilia StålsbyLundborg and Megha Sharma contributed equally for this study and share the last authorship.

'Department of Public Health Sciences, Global Health - Health Systems and

Policy, Karolinska Institutet, 17177 Stockholm, Sweden

${ }^{2}$ Department of Pharmacology, Ruxmaniben Deepchand Gardi Medical

College, Surasa, Ujjain 456006, India

Full list of author information is available at the end of the article
}

(c) The Author(s). 2019 Open Access This article is distributed under the terms of the Creative Commons Attribution 4.0 International License (http://creativecommons.org/licenses/by/4.0/), which permits unrestricted use, distribution, and reproduction in any medium, provided you give appropriate credit to the original author(s) and the source, provide a link to the Creative Commons license, and indicate if changes were made. The Creative Commons Public Domain Dedication waiver (http://creativecommons.org/publicdomain/zero/1.0/) applies to the data made available in this article, unless otherwise stated. 
Table 1 Inpatients' characteristics at surgery departments of the TH and NTH in Madhya Pradesh, India

\begin{tabular}{|c|c|c|c|c|c|c|c|c|c|c|}
\hline & \multicolumn{2}{|l|}{ All patients } & \multicolumn{2}{|l|}{ UUTSI } & \multicolumn{2}{|l|}{ LUTSI } & \multicolumn{2}{|l|}{ RASI } & \multicolumn{2}{|l|}{ EASI } \\
\hline & & NTH, & & NTH, & & & & & & NTH, \\
\hline & $N=6171$ & $N=6263$ & $N=284$ & $N=741$ & $N=748$ & $N=824$ & $N=1394$ & $N=748$ & $N=482$ & $N=763$ \\
\hline & $n(\%)$ & $n(\%)$ & $n(\%)$ & $n(\%)$ & $n(\%)$ & $n(\%)$ & $n(\%)$ & $n(\%)$ & $n(\%)$ & $n(\%)$ \\
\hline \multicolumn{11}{|l|}{ Age in years } \\
\hline $0-17$ & $677(11)^{\mathrm{a}}$ & $377(6)$ & $21(7)$ & $36(5)$ & $45(6)^{a}$ & $15(2)$ & $139(10)^{\mathrm{a}}$ & $26(3)$ & $72(15)$ & $91(12)$ \\
\hline $18-39$ & $2018(33)$ & $2757(44)^{\mathrm{a}}$ & $159(56)$ & $425(57)$ & $69(9)$ & $119(14)$ & $320(23)$ & $281(38)^{a}$ & $239(50)$ & $419(55)$ \\
\hline $40-59$ & $1773(29)$ & $1689(27)$ & $78(27)$ & $197(27)$ & $150(20)$ & 148 (18) & $453(32)$ & $222(30)$ & $108(22)$ & $188(25)$ \\
\hline$\geq 60$ & $1703(28)^{\mathrm{a}}$ & $1440(23)$ & $26(9)$ & $83(11)$ & $484(65)$ & $542(66)$ & $482(35)$ & $219(29)$ & $63(13)$ & $65(8)$ \\
\hline Male & $4601(75)^{\mathrm{a}}$ & $4384(70)$ & $218(77)$ & $546(74)$ & $719(96)$ & $812(99)$ & $1182(85)^{\mathrm{a}}$ & $532(71)$ & $347(72)$ & $610(80)$ \\
\hline Female & $1570(25)$ & $1879(30)^{\mathrm{a}}$ & $66(23)$ & $195(26)$ & $29(4)$ & $12(1)$ & $212(15)$ & $216(29)^{a}$ & $135(28)$ & $153(20)$ \\
\hline Patients prescribed $A B$ & $5430(88)$ & $5402(86)$ & $251(88)$ & $624(84)$ & $748(100)$ & $\begin{array}{l}823 \\
(100)\end{array}$ & $\begin{array}{l}1393 \\
(100)^{\mathrm{a}}\end{array}$ & $628(84)$ & $\begin{array}{l}482 \\
(100)^{\mathrm{a}}\end{array}$ & $719(94)$ \\
\hline Total number of $A B$ prescriptions (Np) & 12,213 & 9598 & 563 & 1055 & 1624 & 1453 & 2767 & 1240 & 1435 & 1679 \\
\hline $\begin{array}{l}\text { Prescriptions adherent to WHOLEM, Np } \\
\text { (percentage of total nr. of prescriptions) }\end{array}$ & $8039(66)^{a}$ & $4019(42)$ & $\begin{array}{l}309 \\
(55)^{\mathrm{a}}\end{array}$ & $346(33)$ & $753(46)^{a}$ & $346(24)$ & $1736(63)^{\mathrm{a}}$ & $510(41)$ & $\begin{array}{l}1054 \\
(73)^{\mathrm{a}}\end{array}$ & $790(47)$ \\
\hline $\begin{array}{l}\text { Prescriptions adherent to NLEMI, Np } \\
\text { (percentage of total nr. of prescriptions) }\end{array}$ & $9119(75)^{\mathrm{a}}$ & $5487(57)$ & $342(61)$ & $592(56)$ & $880(54)^{a}$ & $592(41)$ & $2025(73)^{a}$ & $734(59)$ & $\begin{array}{l}1173 \\
(82)^{\mathrm{a}}\end{array}$ & $984(59)$ \\
\hline $\begin{array}{l}\text { Average length of hospital stay, } \\
\text { days [SD] }\end{array}$ & $9.9[10.0]^{\mathrm{a}}$ & $4.6[3.9]$ & $\begin{array}{l}9.1 \\
{[7.8]^{\mathrm{a}}}\end{array}$ & $3.8[3.2]$ & $\begin{array}{l}13.0 \\
{[11.3]^{\mathrm{a}}}\end{array}$ & $5.0[3.2]$ & $9.8[7.2]^{\mathrm{a}}$ & $5.1[4.0]$ & $\begin{array}{l}11.1 \\
{[9.5]^{\mathrm{a}}}\end{array}$ & $6.3[5.3]$ \\
\hline $\begin{array}{l}\text { Average length of } A B \text { treatment, } \\
\text { days [SD] }\end{array}$ & $9.5[7.6]^{\mathrm{a}}$ & $4.4[3.2]$ & $\begin{array}{l}8.6 \\
{[6.9]^{\mathrm{a}}}\end{array}$ & $3.6[2.6]$ & $11.5[9.0]^{\mathrm{a}}$ & $4.9[3.0]$ & $7.8[5.3]^{\mathrm{a}}$ & $4.4[3.4]$ & $9.0[7.4]^{\mathrm{a}}$ & $5.5[4.0]$ \\
\hline$A B$ prescribed for 1 day & $36(1)$ & $221(7)$ & $7(2)$ & $96(13)$ & $6(1)$ & $44(5)$ & $14(1)$ & $41(5)$ & $9(2)$ & $40(5)$ \\
\hline DDD/100 patient days & 72.5 & $110.5^{\mathrm{a}}$ & 89.5 & $112.3^{\mathrm{a}}$ & 84.6 & $102.1^{\mathrm{a}}$ & 78.3 & $116.1^{\mathrm{a}}$ & 74.9 & $106.0^{\mathrm{a}}$ \\
\hline Total nr. of $A B$ doses administered & 65,288 & 28,600 & 2759 & 2950 & 9415 & 4733 & 13,890 & 4060 & 6270 & 7980 \\
\hline $\begin{array}{l}\text { Total nr. of } A B \text { doses prescribed using } \\
\text { generic names }\end{array}$ & $\begin{array}{l}16,322 \\
(25)^{\mathrm{a}}\end{array}$ & $1716(6)$ & $\begin{array}{l}607 \\
(22)^{\mathrm{a}}\end{array}$ & $118(4)$ & $\begin{array}{l}2542 \\
(27)^{\mathrm{a}}\end{array}$ & $142(3)$ & $4584(33)^{\mathrm{a}}$ & $203(5)$ & $\begin{array}{l}1693 \\
(27)^{\mathrm{a}}\end{array}$ & $399(5)$ \\
\hline $\begin{array}{l}\text { Total nr. of } A B \text { doses } \\
\text { administered parenterally }\end{array}$ & $\begin{array}{l}34,613 \\
(53)\end{array}$ & $\begin{array}{l}19,639 \\
(79)^{\mathrm{a}}\end{array}$ & $\begin{array}{l}1335 \\
(41)\end{array}$ & $\begin{array}{l}2245 \\
(76)^{\mathrm{a}}\end{array}$ & $3672(39)$ & $\begin{array}{l}2603 \\
(55)^{\mathrm{a}}\end{array}$ & $8195(59)$ & $\begin{array}{l}3370 \\
(83)^{\mathrm{a}}\end{array}$ & $\begin{array}{l}4582 \\
(75)\end{array}$ & $\begin{array}{l}7339 \\
(92)^{\mathrm{a}}\end{array}$ \\
\hline
\end{tabular}


Table 2 Classification of surgery inpatients in four selected diagnoses groups, based on their diagnoses

\begin{tabular}{|c|c|c|}
\hline Diagnosis groups (ICD-10 codes) & $\begin{array}{l}\mathrm{TH}, n=2908(100) \\
n(\%)[\%]\end{array}$ & $\begin{array}{l}\text { NTH, } n=3076(100) \\
n(\%)[\%]\end{array}$ \\
\hline Upper urinary tract surgery indications (UUTSI) & $284(10)[100]$ & $741(24)[100]^{\mathrm{a}}$ \\
\hline Kidney/ureter calculi (N20) & 280 [99] & $734[99]^{\mathrm{a}}$ \\
\hline Other & $4[32]$ & $7[1]$ \\
\hline Lower urinary tract surgery indications (LUTSI) & $748(26)[100]$ & $824(27)[100]$ \\
\hline Benign prostate hyperplasia (N40) & $522[70]$ & $558[68]$ \\
\hline Urethral/bladder calculi (N21) & 89 [12] & $142[17]^{\mathrm{a}}$ \\
\hline Urethral stricture (N35) & 85 [11] & $66[8]$ \\
\hline Prostate cancer (C61) & $29[4]$ & $30[4]$ \\
\hline Bladder cancer (C67) & $10[1]$ & $10[1]$ \\
\hline Urethral disease ${ }^{*}$ (N36) & 9 [1] & $10[1]$ \\
\hline Other & $4[<1]$ & $8[<1]$ \\
\hline Routine abdominal surgery indications (RASI) & $1394(48)[100]^{\mathrm{a}}$ & $748(24)[100]$ \\
\hline Hernia (K40-K46) & $965[69]^{\mathrm{a}}$ & $312[42]$ \\
\hline Other gastric/duodenal disease (K31) & $113[8]$ & 151 [20] \\
\hline Biliary calculi (K80) & $73[5]$ & $110[15]$ \\
\hline Anal fissure/fistula (K60) & $103[7]^{a}$ & $30[4]$ \\
\hline Malignancy in Gl-tract (C15-C26) & $78[6]$ & $51[7]$ \\
\hline Cholecystitis (K81) & $30[2]$ & $43[6]$ \\
\hline Anal abscess (K61) & $10[<1]$ & $28[4]$ \\
\hline Other disease in anal region ${ }^{* *}(\mathrm{~K} 62)$ & $10[<1]$ & 8 [1] \\
\hline Colon polyposis (K63.2) & $9[<1]$ & $5[<1]$ \\
\hline Other & $3[<1]$ & $10[<1]$ \\
\hline Emergency abdominal surgery indications (EASI) & $482(17)[100]$ & $763(25)[100]^{\mathrm{a}}$ \\
\hline Appendicitis (K35-K37) & $268[56]$ & $459[60]^{\mathrm{a}}$ \\
\hline Intestinal obstruction (K56) & $132[27]$ & $202[27]$ \\
\hline Peritonitis (K56) & $80[17]$ & 99 [13] \\
\hline Other & $2[<1]$ & $3[<1]$ \\
\hline
\end{tabular}

Numbers of patients with percentages rounded off to the nearest whole number

Abbreviations: $T H$ teaching hospital, NTH non-teaching hospital

"Urethral disease includes fistulas, diverticulosis, carbuncles, and prolepses of the urethra

${ }^{* *}$ Disease in the anal/rectal region includes polyps, stenosis, wounds, bleedings, and prolapses in the anal/rectal region

a Significant $p$-values 